\title{
Chromosome 22q11 in a Xhosa schizophrenia population
}

\author{
Liezl Koen, Dana J H Niehaus, Galen Wright, Louise Warnich, Greetje de Jong, Robin A Emsley, Sumaya Mall
}

Chromosome 22q11 aberrations substantially increase the risk for developing schizophrenia. Although micro-deletions in this region have been extensively investigated in different populations across the world, little is known of their prevalence in African subjects with schizophrenia. We screened 110 African Xhosa-speaking participants with schizophrenia for the presence of micro-deletions. As further verification for the presence or absence of 22q11 microdeletions, we screened 238 Xhosa schizophrenia patients and 240 healthy Xhosa individuals from a larger schizophrenia candidate 22q11 gene study using molecular analyses. Data from molecular and cytogenetic analyses confirmed the absence of 22q11 microdeletions in the Xhosa schizophrenia samples. Although the absence of chromosome 22q11 micro-deletions in this group of patients does not exclude the possibility that it may occur in Xhosa schizophrenia patients, we concluded an extremely low prevalence. Our findings suggest that unique susceptibility loci may be present in this group.

S Afr Med J 2012;102:165-166.
Substantial evidence exists supporting a major contributory role for genes in the causation of schizophrenia, ${ }^{1-3}$ a psychiatric illness characterised by hallucinations, delusions, disorganised thinking, alogia, avolition and other negative symptoms. ${ }^{4}$

A micro-deletion at chromosome $22 \mathrm{q} 11$, the most frequent interstitial deletion found in humans, has been implicated in the onset of schizophrenia. This aberration occurs in approximately 1 in every 4000 live births (a familial type occurs in $<5 \%$ of reported cases) and its occurrence is associated with a variable phenotype. The prevalence of psychosis in those with $22 \mathrm{q} 11$ deletion syndrome is high $(\sim 25 \%)$, suggesting that haploinsufficiency of a gene or genes in this region may confer a substantially increased risk. ${ }^{5}$ It is therefore not surprising that approximately 1 in 4 children with 22q11 deletion syndrome could develop schizophrenia over their lifetime. ${ }^{6}$

The 22q11 micro-deletions have been characterised and mainly involve two areas of $3 \mathrm{Mb}$ ( $87 \%$ of cases) and $1.5 \mathrm{Mb}$ ( $8 \%$ of cases), respectively. The critical overlapping area between these two areas and smaller deletions described in selected cases has led to the identification of the 'schizophrenia critical region' (1.5 Mb in size). This area probably contributes via hemizygous deletion (30 genes located in area) to the development of schizophrenia in approximately $2 \%$ of cases. However it is still uncertain how large the contribution of non-deletion variants in this region may be, especially

Ngaphakathi Workgroup, Department of Psychiatry, Stellenbosch University Tygerberg, W Cape

Liezl Koen, $\mathrm{PhD}$

Dana J H Niehaus, DMed Psychiatry

Robin A Emsley, PhD

Department of Genetics, Stellenbosch University

Galen Wright, BSc Hons

Louise Warnich, $\mathrm{PhD}$

Department of Obstetrics and Gynaecology, Stellenbosch University

Greetje de Jong, $\mathrm{PhD}$

Department of Psychology, Stellenbosch University

Sumaya Mall, MPH if we consider that the PRODH, ZDHHC8 and COMT genes are located in or around this area. ${ }^{6}$

With regard to the $22 \mathrm{q} 11$ micro-deletion, it appears that prevalence differs in different cultural and ethnic groups. For example, Karayiourgou and Gogos ${ }^{6}$ state that '... the lowest rate $(0.3 \%)$ has been reported in a case sample from Japan'. Wiehahn et al. ${ }^{7}$ found that among Afrikaner schizophrenia patients from South Africa, there is a prevalence of $2.4 \%$ of chromosome $22 \mathrm{q} 11$ microdeletions. Phylogenetically, the Afrikaners are closer to European ethnic groups.

However, to date, no cytogenetic studies have focused exclusively on the prevalence of chromosome 22q11 micro-deletions in the black African population. There is a paucity of data on schizophrenia and other mental illnesses in this group. ${ }^{8}$

\section{Aims and objectives}

Our study focused on a Nguni-speaking population group (the Xhosa) who live mainly in the Eastern and Western Cape provinces of South Africa. We aimed to screen for 22q11 micro-deletions in this group to contribute to the genetic profile of an African sample with schizophrenia.

\section{Methods}

The Xhosa people are the second largest and southernmost indigenous African population within South Africa and belong to the Nguni linguistic group. Given the historical and geographical factors that influenced their identity, the Xhosa population can be regarded as representing a culturally homogenous grouping. ${ }^{9}$ Forming part of a larger 'Genetics of Schizophrenia' study, 295 Xhosa schizophrenia patients and 240 healthy Xhosa controls participated in this study. Micro-deletions of the 22q11 locus were analysed using fluorescent in situ hybridisation (FISH) and/ or molecular analyses. Participants provided written, informed consent as approved by the Committee for Human Research of Stellenbosch University, South Africa. All were of Xhosa ethnic origin (4/4 grandparents reported to be of Xhosa origin), while schizophrenia patients additionally met the following criteria: $(i)$ inclusion criteria: (a) structured clinical interview (diagnostic interview for genetic studies, including schedules for the assessment of negative and positive symptoms); (b) diagnosis of schizophrenia or schizo-affective disorder (DSM-IV criteria); and (ii) exclusion criteria: subjects with known medical disorders that may cause psychosis were excluded. 
Table I. Number of Xhosa samples used for the different analyses performed

\begin{tabular}{lllll}
\hline & Total $^{*}$ & FISH analyses & Molecular analyses & Overlap $^{\dagger}$ \\
\hline Xhosa schizophrenia & 295 & 110 & 238 & 53 \\
Xhosa control & 240 & 0 & 240 & 0 \\
& & & & \\
*Total number of unique Xhosa samples. \\
TSamples that were analysed using FISH and also had genotype data available.
\end{tabular}

\section{FISH analyses}

Twenty millilitres of peripheral venous blood was collected from each participant. FISH analyses were performed on a sub-group of the Xhosa schizophrenia patients (i.e. 110 patients, Table I) according to the standardised procedure of the local accredited diagnostic laboratory (UNISTEL), employing the use of the LSI TUPLE 1 spectrum orange probe.

\section{Molecular analyses (Taqman ${ }^{\mathrm{TM}}$ copy number assay)}

As an additional verification for the presence or absence of $22 \mathrm{q} 11$ micro-deletions, data were used from a Xhosa schizophrenia candidate 22q11 gene study (G Wright, 2011 - unpublished data). Genotypes for 14 single nucleotide polymorphisms (SNPs) in the catechol-O-methyltransferase (COMT) gene were available for 238 Xhosa schizophrenia patients (including the 53 of the FISH samples) and 240 healthy Xhosa control individuals (Table I) and were used to identify homozygous samples for all the polymorphisms (Wright, unpublished data). The rationale for this selection criterion is that hemizygous samples, carrying a micro-deletion of this region, would present as homozygotes if conventional genotyping assays are used.

Briefly, a Taqman ${ }^{\mathrm{TM}}$ copy number assay (Applied Biosystems, Foster City, CA, USA) was used to perform a duplex real-time polymerase chain reaction (PCR) including probes for the 22q11 COMT region (Hs01482169_cn) as well as a reference gene (RNAse P). Quadruplicate PCRs were performed for each sample and genomic DNA from a known 22qDS patient was included in every run, serving as a positive control. Reaction conditions, plate setup, PCR cycling and subsequent analysis of the data were performed as described in Drögemöller et al. ${ }^{10}$

\section{Results}

No chromosome 22q11 micro-deletions were detected in the subgroup of samples that underwent FISH analyses. Because of the absence of micro-deletions in these patients, we screened Xhosa samples that had also been analysed for a schizophrenia candidate 22q11 gene study (Table I). Genotype data from this study identified a total of 12 Xhosa samples (from 8 schizophrenia patients and 4 controls) that met the inclusion criterion for duplex real-time PCR analysis and were successfully screened for 22q11 micro-deletions. The 22qDS patient positive control was correctly identified as carrying a single copy of the 22q11 locus in all runs. All the Xhosa samples, however, were predicted to have 2 copies of the 22q11 locus (calculated copy number $1.58-2.19$; confidence $\geq 95 \%$ ) and were therefore negative for micro-deletions in this region.
These data confirm the absence of 22q11 micro-deletions in the Xhosa cohort through molecular and cytogenetic analyses.

\section{Discussion}

To our knowledge, this is the first study exploring the prevalence of chromosome 22q11 micro-deletions in an ethnically homogenous indigenous African sample and its relationship to schizophrenia. We found no deletions of this chromosome in our sample, representing 535 Xhosa individuals (i.e. 295 Xhosa schizophrenia patients and 240 healthy Xhosa controls). We acknowledge that type II errors may have occurred, but at the least this finding suggests an extremely low prevalence of chromosome 22q11 micro-deletions in this population group. Our findings are in contrast to those of Mulle et al., ${ }^{1}$ who identified $29(0.47 \%)$ deletions of the 22q11 region among 6107 schizophrenia patients of Ashkenazi Jewish descent living in the USA.

The lack of any chromosome 22q11 micro-deletion-positive cases means that we cannot comment on any phenotype-genotype relationships. However, given the previously reported low obsessivecompulsive disorder (OCD) prevalence in Xhosa schizophrenic subjects, ${ }^{8}$ this is an intriguing finding. Larger extended studies are needed to detect a low rate of chromosome 22q11 micro-deletions.

This pilot study further raises the possibility that the Xhosa schizophrenia population may have unique susceptibility or protective factors that influence comorbidity patterns in schizophrenia.

\section{References}

1. Mulle JG, Dodd AF, Mscgrath JA, et al. 63 q29 deletions among 7545 schizophrenic subjects. Am J Hum Genet 2010;13:229-236.

2. Greenwood TA, Lazzeroni LC, Murray SS, et al. Analysis of 94 candidate genes and 12 endophenotypes 2. Greenwood TA, Lazzeroni LC, Murray SS, et al. Analysis of 94 candidate genes and 12 endophenotypes
for schizophrenia from the Consortium on the Genetics of Schizophrenia. Am J Psychiatry 2011;168(9):930-946.

3. Cubells JF, Deoreo EH, Harvey PD, et al. Pharmaco-genetically guided treatment of recurrent rage outbursts in an adult male with 15q13.3 deletion syndrome. Am J Med Genet Part A 2011:155:805-810. 4. American Psychiatric Association. The Diagnostic and Statistical Manual of Mental Disorders. 4th ed, text revision (DSM-IV-TR). Washington, DC: American Psychiatric Press, 2000.

Murphy KC, Jones LA, Owen MJC. High rates of schizophrenia in adults with velo-cardio-facial syndrome. Arch Gen Psychiatry 1999;56:940-945.

6. Karayiorgou M, Gogos JA. The molecular genetics of the 22q11 associated schizophrenia. Brain Res Mol Brain Res 2004;20:95-104.

Wiehahn GJ, Bosch GP, Du Preez RR, et al. Assessment of the frequency of the 22q11 deletion in Afrikaner schizophrenic patients. Am J Med Genet 2004;15:20-22.

8. Niehaus DJ, Koen L, Muller J, et al. Obsessive compulsive disorder prevalence in Xhosa speaking Niehaus DJ, Koen L, Muller J, et al. Obsessive compuls
schizophrenia patients. S Afr Med J 2005;95(2):120-122.

9. Mostert N. Frontiers: The Epic of South Africass Creation and the Tragedy of the Xhosa people. New York: Knopf, 1992

10. Drögemöller BI, Wright GEB, Niehaus DJH, et al. Characterization of the genetic profile of CYP2C19 in two South African populations. Pharmacogenomics 2010;11(8):1095-1103. 